\title{
A CLASS OF INTEGRAL EQUATIONS WHICH GENERALIZE ABEL'S EQUATION
}

\section{EDMUND PINNEY}

Introduction. A general class of integral equations is solved in $\$ 1$. Specific examples are given in $\$ 3$ which are shown in $\$ 5$ to specialize to an equation from which Abel's may readily be derived. In this sense the results of this paper generalize Abel's integral equation solution. Another interesting special case is given in $\S 6$.

1. A general theorem. Our development depends on this theorem:

THEOREM. (i) Let the function $g(x)$ possess a continuous derivative for $x>0$, and let $\int^{\infty} g(t) t d t$ converge. Let $p(x), q(x)$ be functions for which

$$
\int_{0}^{\infty} \int_{0}^{\infty}\left|g\left(\left(x^{2}+s^{2}+t^{2}\right)^{1 / 2}\right)\right||p(s)||q(t)| d s d t \text { exists, }
$$

and for which the condition

$$
\int_{0}^{\pi / 2} p(r \cos \theta) q(r \sin \theta) d \theta=1
$$

holds. Then

$$
\phi(x)=f(x) \equiv-\frac{1}{x} \frac{d}{d x} \int_{0}^{\infty} g\left(\left(x^{2}+t^{2}\right)^{1 / 2}\right) q(t) d t
$$

is a solution of the integral equation

$$
g(x)=\int_{0}^{\infty} \phi\left(\left(x^{2}+t^{2}\right)^{1 / 2}\right) p(t) d t .
$$

(ii) If $\phi(x)$ is a solution of (4) having a continuous derivative for $x>0$, such that $\int^{\infty} \phi(t) t d t$ converges and such that

$$
\int_{0}^{\infty} \int_{0}^{\infty}\left|\phi\left(\left(x^{2}+s^{2}+t^{2}\right)^{1 / 2}\right)\right||p(s)||q(t)| d s d t \text { exists, }
$$

then

$$
\phi(x)=f(x) .
$$

Proof. From (3),

Received by the editors June 5, 1944, and, in revised form, September 18, 1944. 


$$
\begin{aligned}
I & \equiv \int_{0}^{\infty} f\left(\left(x^{2}+t^{2}\right)^{1 / 2}\right) p(t) d t \\
& =-\frac{1}{x} \frac{d}{d x} \int_{0}^{\infty} p(t) d t \int_{0}^{\infty} g\left(\left(x^{2}+t^{2}+s^{2}\right)^{1 / 2}\right) q(s) d s .
\end{aligned}
$$

By (1) and Fubini's theorem this may be written

$$
I=-\frac{1}{x} \frac{d}{d x} \int_{0}^{\infty} g\left(\left(x^{2}+r^{2}\right)^{1 / 2}\right) r d r \int_{0}^{\pi / 2} p(r \cos \theta) q(r \sin \theta) d \theta .
$$

From (2) and the conditions on $g(x),{ }^{1}$

$$
\begin{aligned}
I & =-\frac{1}{x} \frac{d}{d x} \int_{0}^{\infty} g\left(\left(x^{2}+r^{2}\right)^{1 / 2}\right) r d r \\
& =-\int_{0}^{\infty} g^{\prime}\left(\left(x^{2}+r^{2}\right)^{1 / 2}\right) \frac{r d r}{\left(x^{2}+r^{2}\right)^{1 / 2}}=-\left[g\left(\left(x^{2}+r^{2}\right)^{1 / 2}\right)\right]_{0}^{\infty} \\
& =g(x) .
\end{aligned}
$$

Therefore $f(x)$ is a solution of (4).

If $\phi(x)$ is a solution of (4) as specified in (ii), then exactly as we showed that $I=g(x)$ we may show that $f(x)=\phi(x)$, completing the proof.

2. The functions $p(x)$ and $q(x)$. The application of this theorem requires the determination of pairs of functions $p(x), q(x)$ satisfying (2). We shall now consider the set of such pairs for which $p(x), q(x)$ are both continuous and for which

$$
\int_{0}^{\infty} e^{-z t^{2}}|p(t)| d t \text { and } \int_{0}^{\infty} e^{-z t^{2}}|q(t)| d t \quad \text { converge, } z>0 .
$$

By (2), for $z>0$,

$$
\int_{0}^{\infty} e^{-z r^{2}} \int_{0}^{\pi / 2} p(r \cos \theta) q(r \sin \theta) d \theta r d r=\int_{0}^{\infty} e^{-z r^{2}} r d r
$$

By Fubini's theorem and (6) we may transform from polar to rectangular coordinates, obtaining

$$
P(z) Q(z)=1 / 2 z,
$$

where

$$
P(z)=\int_{0}^{\infty} e^{-z t^{2}} p(t) d t, \quad Q(z)=\int_{0}^{\infty} e^{-z t^{2}} q(t) d t .
$$

\footnotetext{
${ }^{1}$ Whittaker and Watson, Modern analysis, Cambridge University Press, §4.44.
} 
By Lerch's theorem on null functions, ${ }^{2}$ if $p(x)$ and $q(x)$ satisfy (7) and (8), they also satisfy (2).

We note that (7) is symmetrical in $P$ and $Q$, so we conclude that $p$ and $q$ may be interchanged throughout the theorem of $\$ 1$.

3. Special values of $p(x)$ and $q(x)$. We now concern ourselves with finding pairs of functions $p(t), q(t)$ satisfying (7), (8) and which may be used in the theorem of $\$ 1$. In this regard we note an extended Laguerre function definition,

$$
L_{\nu}^{\mu}(z)=-\frac{\sin \pi \nu}{\pi} \Gamma(\mu+\nu+1) \sum_{0}^{\infty} \frac{\Gamma(p-\nu)}{\Gamma(p+\mu+1)} \frac{z^{p}}{p !} .
$$

This reduces to the Laguerre polynomials when $\nu$ is a non-negative integer $n$. It is related to the Whittaker function ${ }^{3} M_{m, n}$ by the relation

$$
L_{\nu}^{\mu}(z)=\frac{\Gamma(\mu+\nu+1)}{\Gamma(\mu+1) \Gamma(\nu+1)} z^{-\mu / 2-1 / 2} e^{z / 2} M_{\mu / 2+\nu+1 / 2, \mu / 2}(z)
$$

and to the Bessel function $J_{\mu}$ by

$$
\lim _{s \rightarrow 0}\left(\frac{s}{\nu}\right)^{\mu} L_{\nu / 8}^{\mu}(s z)=(\nu z)^{-\mu / 2} J_{\mu}\left(2(\nu z)^{1 / 2}\right)
$$

uniformly in $\nu z$ for $\nu z$ bounded. ${ }^{4}$

From (10) and $\$ 16.4$ of Whittaker and Watson, for $|z| \gg 1$,

$$
L_{\nu}^{\mu}(z) \sim-\frac{\sin \pi \nu}{\pi} \Gamma(\mu+\nu+1) z^{-\mu-\nu-1} e^{z}+\frac{e^{ \pm \pi i \nu}}{\Gamma(\nu+1)} z^{\nu}
$$

where the upper or lower sign is taken according as $I(z) \lessgtr 0$.

In view of this we may expand the Laguerre functions and integrate term by term, getting, for $R(z)>R(k)$,

$$
\begin{aligned}
& \int_{0}^{\infty} e^{-z t^{2}} t^{1-2 \mu} L_{\nu}^{-\mu}\left(k t^{2}\right) d t=\frac{\Gamma(1-\mu+\nu)}{2 \Gamma(1+\nu)} z^{\mu-\nu-1}(z-k)^{\nu}, R(\mu)<1, \\
& \int_{0}^{\infty} e^{-z t^{2}} t^{2 \mu-1} L_{-\nu}^{\mu-1}\left(k t^{2}\right) d t=\frac{\Gamma(\mu-\nu)}{2 \Gamma(1-\nu)} z^{-\mu+\nu}(z-k)^{-\nu}, R(\mu)>0 .
\end{aligned}
$$

Therefore, from (7), (8), we may take

${ }^{2}$ Acta Math. vol. 27 (1903) pp. 339-352.

${ }^{3}$ Whittaker and Watson, Modern analysis, Cambridge University Press, chap. 16.

${ }^{4}$ G. Szegö, Orthogonal polynomials, Amer. Math. Soc. Colloquium Publications, vol. 23, New York, 1939, Theorem 8.1.3. 


$$
\begin{aligned}
& p(t)=C t^{1-2 \mu} L_{\nu}^{-\mu}\left(k t^{2}\right), \\
& q(t)=\frac{2 \nu}{C} \frac{\sin \pi(\mu-\nu)}{\sin \pi \nu} t^{2 \mu-1} L_{-\nu}^{\mu-1}\left(k t^{2}\right),
\end{aligned}
$$

where $0<R(\mu)<1$, and $C, k$ are arbitrary except that $C \neq 0$.

(13) may be specialized in various ways. In particular from (11) and the fact that

$$
L_{\nu}^{\mu}(z)=\frac{\sin \pi \nu}{\sin \pi(\mu+\nu)} e^{z} L_{-\mu-\nu-1}^{\mu}(-z),
$$

we may have

$$
\begin{aligned}
& p(t)=C t^{1-\mu} J_{-\mu}(\alpha t), \\
& q(t)=(\alpha / C) t^{\mu} I_{\mu-1}(\alpha t),
\end{aligned}
$$

for $0<R(\mu)<1$.

Letting $\nu \rightarrow 0$ in (13) we have, when $0<R(\mu)<1$,

$$
\begin{aligned}
p(t) & =t^{1-2 \mu}, \\
q(t) & =(2 / \pi) \sin \pi \mu t^{2 \mu-1} .
\end{aligned}
$$

Abel's integral equation may be derived from that corresponding to this $p$ and $q$.

When $\nu=n$, an integer not less than 0 , the Laguerre function in $p(t)$ in (13) becomes a Laguerre polynomial. By (9) we may write for (13), for $0<R(\mu)<1$,

$$
\begin{aligned}
& p(t)=C t^{1-2 \mu} L_{\nu}^{-\mu}\left(k t^{2}\right), \\
& q(t)=-\frac{2}{C} \frac{\Gamma(\nu+1)}{\Gamma(\mu) \Gamma(\nu-\mu+1)} t^{2 \mu-1} F\left(\nu ; \mu ; k t^{2}\right) .
\end{aligned}
$$

Therefore for $0<R(\mu)<1$,

$$
\begin{aligned}
& p(t)=C t^{1-2 \mu} L_{n}^{-\mu}\left(k t^{2}\right), \\
& q(t)=-\frac{2}{C} \frac{n !}{\Gamma(\mu) \Gamma(n-\mu+1)} t^{2 \mu-1} F\left(n ; \mu ; k t^{2}\right) .
\end{aligned}
$$

A special case of interest is that in which $\mu=1 / 2$ in (15). Then we have

$$
\begin{aligned}
& p(t)=C \cos \alpha t \\
& q(t)=(2 / \pi C) \operatorname{ch} \alpha t .
\end{aligned}
$$


4. A counter example. (1) is a severe restriction when $p(t), q(t)$ have the general values given in (13), (15). Although the condition might be weakened somewhat it appears unlikely that it could be weakened very much. The following example, very kindly pointed out to me by Professor Harry Bateman, illustrates a case in which our theorem does not hold, despite the fact that $g(x) \rightarrow 0$ exponentially as $x \rightarrow \infty$.

Sonine ${ }^{5}$ showed that for $a>c>0,0<n<p+1>3 / 2$,

$$
\begin{aligned}
\int_{0}^{\infty} J_{p-1}\left(c\left(x^{2}+t^{2}\right)^{1 / 2}\right)\left(x^{2}+t^{2}\right)^{-p / 2-1 / 2} J_{n-1}(a t) t^{n} d t \\
=\frac{(c / 2)^{p-1}}{\Gamma(p)} x^{n-1} K_{n-1}(a x) .
\end{aligned}
$$

Therefore when $0<n<1$ and

$$
\phi(z)=J_{p-1}(c z) z^{-p-1}
$$

and

$$
g(z)=\frac{(c / 2)^{p-1}}{\Gamma(p)} z^{n-1} K_{n-1}(a z),
$$

for the theorem to hold it is necessary that

$$
\begin{array}{r}
\phi(x)=-\frac{a}{x} \frac{d}{d x}\left\{\frac{(c / 2)^{p-1}}{\Gamma(p)} \int_{0}^{\infty}\left(x^{2}+t^{2}\right)^{n / 2-1 / 2} K_{n-1}\left(a\left(x^{2}+t^{2}\right)^{1 / 2}\right)\right. \\
\left.\cdot I_{-n}(a t) t^{1-n} d t\right\} .
\end{array}
$$

This, however, is impossible since $p$ appears only in the factor $(c / 2)^{p-1} / \Gamma(p)$. Therefore the theorem fails. However it is easy to see that the integral in (1) does not exist.

5. Abel's equation. In this section we shall establish the relation between the integral equation corresponding to (16) and Abel's integral equation. The former and its solution are

$$
\begin{aligned}
g(x) & =\int_{0}^{\infty} \phi\left(\left(x^{2}+t^{2}\right)^{1 / 2}\right) t^{1-2 \mu} d t \\
\phi(x) & =-\frac{2}{\pi} \sin \pi \mu \frac{1}{x} \frac{d}{d x} \int_{0}^{\infty} g\left(\left(x^{2}+t^{2}\right)^{1 / 2}\right) t^{2 \mu-1} d t,
\end{aligned}
$$

provided $0<R(\mu)<1, \int_{0}^{\infty} \int_{0}^{\infty}\left|g\left(\left(x^{2}+t^{2}+s^{2}\right)^{1 / 2}\right)\right| t^{1-2 \mu} s^{2 \mu-1} d s d t$ exists, and $\int^{\infty} g(t) t d t$ converges.

S Math. Ann. vol. 16 (1880) pp. 50, 51. 
Write

In (20) let

$$
\begin{aligned}
& \Phi(x)=x^{\mu-2} \phi\left(x^{-1 / 2}\right), \\
& G(x)=2 x^{-\mu} g\left(x^{-1 / 2}\right) .
\end{aligned}
$$

so

$$
s=1 /\left(x^{2}+t^{2}\right),
$$

$$
t^{2}=x^{2} s^{-1}\left(x^{-2}-s\right), \quad 2 t d t=-s^{-2} d s .
$$

Then

$$
2 g(x)=\int_{0}^{x^{-2}} \phi\left(s^{-1 / 2}\right) s^{\mu-2} x^{-2 \mu}\left(\frac{1}{x^{2}}-s\right)^{-\mu} d s
$$

implies that

$$
\phi(x)=-\frac{1}{\pi} \sin \pi \mu \frac{1}{x} \frac{d}{d x} \int_{0}^{x^{-}} g\left(s^{-1 / 2}\right) s^{-\mu-1} x^{2 \mu-2}\left(\frac{1}{x^{2}}-s\right)^{\mu-1} d s .
$$

That is,

$$
G(x)=\int_{0}^{x} \Phi(s)(x-s)^{-\mu} d s
$$

implies that

$$
\Phi(x)=\frac{1}{\pi} \sin \pi \mu x^{\mu} \frac{d}{d x}\left\{x^{1-\mu} \int_{0}^{x} G(s)(x-s)^{\mu-1} \frac{d s}{s}\right\} .
$$

This is valid only if $G(0)=0$, which we shall assume to be the case at present. Replace $s$ by $s x$. Then

$$
\begin{aligned}
\Phi(x) & =\frac{1}{\pi} \sin \pi \mu x^{\mu} \frac{d}{d x} \int_{0}^{1} G(s x)(1-s)^{\mu-1} \frac{d s}{s} \\
& =\frac{1}{\pi} \sin \pi \mu x^{\mu} \int_{0}^{1} G^{\prime}(s x)(1-s)^{\mu-1} d s,
\end{aligned}
$$

or

$$
\Phi(x)=\frac{1}{\pi} \sin \pi \mu \int_{0}^{x} G^{\prime}(s)(x-s)^{\mu-1} d s .
$$

Suppose now that $G(0) \neq 0$. Assume $G(0)$ finite. Write $\Phi(x)$ as the solution, assumed to exist, of

$$
G(x)=\int_{0}^{x} \Phi(s)(x-s)^{-\mu} d s .
$$

Now $G_{1}(x)=G(x)-G(0)$ satisfies our previous conditions and therefore has a solution $\Phi_{1}(x)$ of the equation 


$$
G_{1}(x)=\int_{0}^{x} \Phi_{1}(s)(x-s)^{-\mu} d s
$$

which is given by

$$
\Phi_{1}(x)=\frac{1}{\pi} \sin \pi \mu \int_{0}^{x} G^{\prime}(s)(x-s)^{\mu-1} d s .
$$

Suppose we write $\Phi_{1}(x)=\Phi(x)-\sigma(x)$. Then

$$
G(0)=\int_{0}^{x} \sigma(s)(x-s)^{-\mu} d s .
$$

This is readily seen to have the solution

Now

$$
\sigma(x)=\frac{1}{\pi} \sin \pi \mu G(0) x^{\mu-1} .
$$

$$
\Phi(x)=\Phi_{1}(x)+\sigma(x)
$$

so the equation

$$
G(x)=\int_{0}^{x} \Phi(s)(x-s)^{-\mu} d s, \quad 0<R(\mu)<1,
$$

has the solution

$$
\Phi(x)=\frac{1}{\pi} \sin \pi \mu\left\{G(0) x^{\mu-1}+\int_{0}^{x} G^{\prime}(s)(x-s)^{\mu-1} d s\right\} .
$$

The existence of this solution is obvious. This result is Abel's equation. It can be transformed into its most general form by a linear transformation on $x$.

6. An interesting special case. We note that the equation corresponding to (19) may readily be transformed to

$$
g(x)=\int_{x}^{\infty} \phi(u) \frac{\cos \left(\alpha(u-x)^{1 / 2}\right)}{(u-x)^{1 / 2}} d u
$$

which implies a solution

$$
\phi(x)=-\frac{1}{\pi} \frac{d}{d x} \int_{x}^{\infty} g(u) \frac{\operatorname{ch}\left(\alpha(u-x)^{1 / 2}\right)}{(u-x)^{1 / 2}} d u
$$

provided $\int_{0}^{\infty} \int_{0}^{\infty}\left|g\left(x^{2}+s^{2}+t^{2}\right)\right||\cos \alpha s| \operatorname{ch} \alpha t d s d t$ exists.

Massachusetts Institute of Technology 